\title{
Las lecciones aprendidas del estudio ORBITA: ¿Cuáles lecciones?
}

\author{
Lessons learn from ORBITA trial: Which lessons?
}

Revista Argentina de Cardioangiología Intervencionista 2018;9(2):83-84. Doi: 10.30567/RACI/201802/0083-0084

En enero 4 del 2018 fue publicado en el Lancet el estudio aleatorizado ORBITA, que comparó angioplastia coronaria (PCI) con implante de stents liberadores de fármacos (DES) vs. tratamiento médico óptimo (TMO), pero con un sham procedure realizado en la rama de TMO: es decir, a todos los pacientes se les efectuó coronariografía (CCG) y, en forma ciega, a unos se les realizó PCI con DES y a los otros solo CCG, y en todos las lesiones fueron evaluadas con medición de flujo de reserva coronario imitando que se le hacía una PCI, esto es, sham procedure o placebo. Previamente todos los pacientes tenían CCG diagnóstica que evidenciaba una lesión severa $(\geq 70 \%)$ en un vaso epicárdico principal, de acuerdo con los criterios de inclusión del estudio.

Los puntos finales se refirieron a la diferencia en tolerancia al ejercicio pre- y postratamiento. La diferencia entre ambos grupos PCI vs. placebo fue de 0,16 segundos $(\mathrm{p}=0.200)$, aunque los cambios entre basal y postratamiento fueron más favorables en el grupo PCI comparado con el placebo. Obviamente, estas últimas diferencias no fueron significativas dado el pequeño tamaño de la muestra. ${ }^{1-3}$

Si bien ni en el análisis principal ni en el material suplementario (de lectura obligatoria en estos estudios) se informa cuántos pacientes fueron inicialmente analizados y/o CCG realizadas por sospechas de enfermedad coronaria (EC) estable y/o inestable, se podría asumir, de manera conservadora, que en cada uno de los 5 importantes centros de Gran Bretaña participantes se realizaron 1000 procedimientos por año (número igual al que realiza nuestro grupo CECI). Y que, al abarcar el período de reclutamiento de diciembre de 2013 a julio de 2017, se podría estimar que en estos 5 centros se efectuaron aproximadamente 18040 procedimientos de los cuales se incluyeron 200 pacientes. Es decir, el 1,1\% de los pacientes con sospecha de EC a quienes se les realizó CCG en esos centros fue finalmente incluido y aleatorizado.

Estos números, $1,1 \%$, no se han reportado en el estudio y si bien son cifras estimativas, probablemente las diferencias sean mayores, ya que dadas las características de la Salud Pública de Gran Bretaña, de la que soy un gran admirador, el número de 1000 procedimientos por año parece extremadamente conservador.

Entonces podríamos decir que los hallazgos del ORBITA podrían ser extrapolados al 1\% de la población con EC severa, lo cual se debería haber puesto de manifiesto en todas las comunicaciones que se hicieron públicas usando tanto las redes sociales como el periodismo escrito y televisivo que motivó esta publicación.

Ahora bien, los criterios de inclusión eran lesiones angiográficas severas $\geq 70 \%$ por estimación visual y los autores muestran en el material suplementario imágenes, naturalmente estáticas, de las lesiones severas de esos 200 pacientes. Si uno analiza cada una de estas imágenes, con el error obvio de ser imágenes estáticas aunque es de imaginar que para la publicación se eligieron las que angiográficamente eran las más relevantes, nos encontramos que, a mi criterio, 82 de ellas (41\%) eran o lesiones intermedias $<70 \%$ (que era una causa de exclusión), o lesiones en vasos secundarios como ramas póstero-ventriculares de arteria $\mathrm{Cx}$ o de $\mathrm{CD}$ de pequeñísimo tamaño y/o obstrucciones severas muy distales de ramos epicárdicos principales. ${ }^{2}$

Desde los estudios originales del CASS, hace muchos años, sabemos que el análisis crítico de la anatomía coronaria y de la CCG tiene una gran relevancia para evaluar correctamente el pronóstico de la cardiopatía isquémica, cuando se hace una lectura adecuada de la misma ${ }^{4,5}$.

El estudio ERACI IV ${ }^{6-7}$ mostró que la incidencia de eventos adversos en lesiones de este tipo no tratadas con PCI más DES y dejadas solo con TMO a 3 años fue de 3,9\% de nuevas revascularizaciones sin registrarse ningún evento adverso clínico mayor como muerte o infarto de miocardio; de hecho, el score de riesgo ERACI no las incluyó ni en forma basal y/o residual luego de la PCI. ${ }^{8}$

Es decir, no solo estamos hablando del 1\% de la población con EC sino también que el $41 \%$ de ellos tendría demostrada buena evolución con TMO, de manera que estamos discutiendo por el 0,6\% de pacientes con EC severa que llegaría a nuestra consulta. 
Sin embargo, esto no termina acá, recientemente nos enteramos que el $85 \%$ de los pacientes no tratados con PCI se realizaron finalmente angioplastia?.

Después de 25 años de haberse publicado los primeros estudios aleatorizados entre cirugía coronaria, TMO y PCI en pacientes con EC severa, es muy difícil para todos los que participamos en esas experiencias observar cómo se quiere minimizar o desacreditar los claros beneficios de la PCI en determinados grupos de pacientes ${ }^{10-12}$.

El estudio ORBITA solo nos debe enseñar que cuando leamos resultados similares a estos, debemos estar muy alertas antes de comentarlos y/o aceptarlos, y responder adecuadamente con un análisis detallado de su metodología y analizando en profundidad los criterios científicos utilizados para obtener esos hallazgos.

Aunque la angioplastia coronaria tiene, sin duda, conocidas limitaciones, ninguna de ellas fue o es que carezca de "sentido común" o que vaya en desmedro del beneficio de nuestros pacientes.

\section{Alfredo E. Rodríguez MD, PhD, FACC, FSCAI}

Editor en Jefe de RACI

\section{BIBLIOGRAFÍA}

1. Al-Lamee $R$, Thompson D, Dehbi HM, et al. Percutaneous coronary intervention in stable angina (ORBITA): a double-blind, randomised controlled trial. Lancet 2018;391(10115):31-40.

2. Al-Lamee $R$, Thompson D, Dehbi HM, et al. Percutaneous coronary intervention in stable angina (ORBITA): a double-blind, randomised controlled trial. Lancet 2018;391(10115):31-40. Supp Material.

3. De Bruyne B, Fearon WF, Pijls NH, et al; FAME 2 Trial Investigators. Fractional flow reserve-guided $P C I$ for stable coronary artery disease. N Engl J Med 2014;371:1208-17.

4. MockMB, Ringqvist I, Fisher $L D$, et al. Survival of medically treated patients in the coronary artery surgery study (CASS) registry. Circulation 1982 Sep;66(3):562-8.

5. Rodríguez AE. Valor pronóstico de la coronariografía: dónde está el error. Revista de la Federación Argentina de Cardiología (FAC)1987, VOL 1:13.

6. Rodríguez AE, Santaera O, Larribau M, et al; ERACI IV Investigators. Second versus First Drug Eluting Stents in Complex lesions Subsets: 3 Years Follow Up of ERACIIV Stuydy. Minerva Cardioangiol 2017 Feb;65(1):81-90.

7. Rodríguez AE, Fernández-Pereira C, Mieres J, Mendoza J, Sartori F. Can We Improve the Outcomes of Multivessel Disease Using Modified SYNTAX and Residual SYNTAX Scores? Curr Cardiol Rep 2017:20:1-7.
8. Rodríguez AE, Fernández-Pereira C, Mieres J, Santaera O, Antoniucci D; ERACI IV investigators. Modifying angiographic syntax score according to PCI strategy: lessons learn from ERACI IV Study. Cardiovasc Revasc Med 2015 Oct-Nov; 16(7):418-20.

9. ORBITA Continues to Surprise: $85 \%$ of Sham Arm Opted for PCI When Trial Ended. Controversies in interventional cardiology l: interventional management in stable coronary artery disease. Presented at: SCAI 2018. April 25, 2018. San Diego, CA.

10. Parisi AF, Folland ED, Hartigan P. A comparison of angioplasty with medical therapy in the treatment of single-vessel coronary artery disease. Veterans Affairs ACME Investigators. N Engl J Med 1992;236:10-6.

11. Coronary angioplasty versus medical therapy for angina: the second Randomised Intervention Treatment of Angina (RITA-2) trial. RITA-2 trial participants. Lancet 1997;350:461-8.

12. Daemen J, Boersma E, Flather $M$, et al. Long-term safety and efficacy of percutaneous coronary intervention with stenting and coronary artery bypass surgery for multivessel coronary artery disease: a meta-analysis with 5-year patient-level data from the ARTS, ERACI-II, MASS-II, and SoS trials. Circulation 2008;118:1146-54. 